\title{
Estimating the intermonth covariance between rainfall and the atmospheric circulation
}

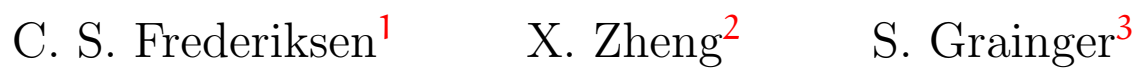

(Received 27 January 2011; revised 31 May 2011)

\begin{abstract}
The seasonal mean of a climate variable consists of a slow and intraseasonal component. Existing methods for deriving coupled patterns between intraseasonal components assume stationarity and first order autoregressive processes. This does not hold for a variable such as rainfall where the daily data consists of dichotomous (on/off) events. It is possible to formulate a more general method for such two-state climate variables but it requires an estimate of the intermonth covariance. We use a stochastic two-state first-order Markov chain model fitted to daily Australian rainfall data to provide an estimate of the intermonth covariance with daily $500 \mathrm{hPa}$ atmospheric geopotential height anomalies. We show that the estimate of the intermonth covariance is much smaller than the within-month covariance between rainfall and the $500 \mathrm{hPa}$ height intraseasonal component.
\end{abstract}

http://anziamj . austms.org.au/ojs/index.php/ANZIAMJ/article/view/3927 gives this article, (c) Austral. Mathematical Soc. 2011. Published June 6, 2011. ISSN 1446-8735. (Print two pages per sheet of paper.) Copies of this article must not be made otherwise available on the internet; instead link directly to this URL for this article. 


\section{Contents}

1 Introduction

C191

2 Methodology

C193

2.1 Statistical model for monthly mean fields . . . . . . . . . C193

2.2 Estimating the intermonth covariance with rainfall . . . . .

C195

3 Example

C198

4 Conclusions

C200

References

C203

\section{Introduction}

The interannual variability of surface climate fields, such as seasonal mean temperature and rainfall, are generally related to the interannual variability of local or hemispheric seasonal mean atmospheric pressure fields. This relationship is quantifiable by the interannual covariance between the two fields. Interannual variability in the seasonal means of climate variables arise from sources which are categorized as: (a) slowly varying (interannual and longer) external forcing (for example, sea surface temperature forcing) and internal dynamics; and (b) internal dynamics within the season [1]. Zheng and Frederiksen [2] conceptualised the seasonal mean of a climate variable as consisting of two components, referred to as the slow and intraseasonal components. The former is regarded as potentially predictable because it is associated with slowly varying external and internal processes, and the latter is essentially unpredictable. A number of studies showed that skilful statistical seasonal forecast schemes can be derived from a knowledge of the behaviour of the slow component $[3,4,5]$.

For the seasonal prediction of surface climate fields, a knowledge of the spatial 
patterns relating the slow component of the seasonal mean pressure fields to the slow component of the surface climate variable should provide a better understanding of the meteorological phenomena associated with forecast skill. Conversely, the spatial patterns that relate the intraseasonal components of the pressure field and the surface climate variable should be related to the uncertainty in forecast skill $[1,4]$. To derive these coupled patterns in the two components of the two variables requires an estimate of the covariance matrices for each component. When data is available at sub-seasonal timescales (for example, daily or monthly) it has been shown that under certain assumptions an estimate of the covariance of the intraseasonal components is able to be made $[1,2,4,5]$. The covariance of the slow components is then estimated as the difference between the covariance of the original pair of variables, using moment estimation, and the covariance of the intraseasonal components.

A common assumption when estimating the covariance between intraseasonal components is that the monthly statistics of the intraseasonal components are stationary and the underlying daily time series are first order autoregressive processes (AR1). The intermonth covariance between the pairs of climate variables is also assumed to be stationary and constant $[1,4,6]$. These assumptions do not hold, in general, for a variable such as rainfall where the daily data consists of dichotomous (on/off) events [7]. It is possible to formulate a more general method for such two state climate variables but it requires an estimate of the intermonth covariance. To provide such an estimate for all seasons, we use a stochastic, two state, first order, Markov chain model fitted to daily Australian rainfall data and daily $500 \mathrm{hPa}$ atmospheric geopotential height anomalies. 


\section{Methodology}

\subsection{Statistical model for monthly mean fields}

In order to estimate the covariance matrix of the intraseasonal components, data needs to be available at least as monthly averages within the season $[1,2]$. It is then useful to think of the monthly means of a climate variable $x$ as a statistical random variable $[1,2]$ consisting of two components:

$$
x_{y m}=\mu_{y}+\varepsilon_{y m} .
$$

Here, $x_{y m}$ represent monthly anomalies of a climate variable, with the annual cycle removed, in year $y(=1, \ldots, Y)$, month $m(=1,2,3$, corresponding, for example, to March, April, May, respectively); $\mu_{y}$ represents the slow, or more potentially predictable component, assumed constant over each month in the season; $\varepsilon_{y m}$ is the intraseasonal, or essentially unpredictable, component. The vector $\left(\varepsilon_{y 1}, \varepsilon_{y 2}, \varepsilon_{y 3}\right)$ is assumed to comprise a stationary and independent random vector with respect to year. Equation (1) implies that month-to-month fluctuations, or intraseasonal variability, arise entirely from this component (for example, $x_{y 1}-x_{y 2}=\varepsilon_{y 1}-\varepsilon_{y 2}$ ). We use the convention that an average over any index will be represented by a circle. For example, $x_{y \circ}$ is an average over all months in the season, and $x_{\circ \circ}$ is an average over all months and years. That is,

$$
x_{y o}=\frac{1}{3} \sum_{m=1}^{3} x_{y m}=\mu_{y}+\varepsilon_{y o} .
$$

Thus, $\mu_{y}$ is also thought of as the seasonal population mean and $\varepsilon_{y \circ}$ is a residual, associated with predominantly intraseasonal weather noise [1, 2], which is assumed to have zero expectation over all years $Y$. That is,

$$
E\left[\varepsilon_{y o}\right] \equiv \frac{1}{Y} \sum_{y=1}^{Y} \varepsilon_{y o}=0,
$$


where $E$ represents the expectation value, defined as the average value over all years. In this article, pairs of climate variables $\left(x_{y m}, x_{y m}^{\prime}\right)=\left(\mu_{y}+\varepsilon_{y m}, \mu_{y}^{\prime}+\right.$ $\left.\varepsilon_{y m}^{\prime}\right)$ are considered.

The covariance $\mathrm{V}\left(\varepsilon_{y_{0}}, \varepsilon_{y_{0}}^{\prime}\right)$ of the seasonal mean of the intraseasonal components of two climate variables $\left(x_{y_{0}}, x_{y_{0}}^{\prime}\right)$ is estimated by

$$
\begin{aligned}
V\left(\epsilon_{y \circ}, \varepsilon_{y \circ}^{\prime}\right) & =E\left[\frac{1}{9} \sum_{m=1}^{3} \varepsilon_{y m} \sum_{n=1}^{3} \epsilon_{y n}^{\prime}\right] \\
& =\frac{1}{9}\left(\sigma_{11}+\sigma_{22}+\sigma_{33}+\sigma_{12}+\sigma_{21}+\sigma_{23}+\sigma_{32}+\sigma_{13}+\sigma_{31}\right)
\end{aligned}
$$

where $\sigma_{\mathrm{mn}}=\mathrm{E}\left[\epsilon_{y \mathrm{~m}} \epsilon_{y \mathfrak{n}}^{\prime}\right]$ is the covariance between the two intraseasonal components in months $m$ and $n$. There are nine terms that need to be estimated $\left(\sigma_{11}, \sigma_{22}, \sigma_{33}, \sigma_{12}, \sigma_{21}, \sigma_{23}, \sigma_{32}, \sigma_{13}, \sigma_{31}\right)$. It follows also from (1) that

$$
\begin{aligned}
& E\left[\left(\begin{array}{c}
\varepsilon_{y 1}-\varepsilon_{y 2} \\
\varepsilon_{y 2}-\varepsilon_{y 3}
\end{array}\right)\left(\begin{array}{c}
\varepsilon_{y 1}^{\prime}-\varepsilon_{y 2}^{\prime} \\
\varepsilon_{y 2}^{\prime}-\varepsilon_{y 3}^{\prime}
\end{array}\right)^{\top}\right]=E\left[\left(\begin{array}{l}
x_{y 1}-x_{y 2} \\
x_{y 2}-x_{y 3}
\end{array}\right)\left(\begin{array}{c}
x_{y 1}^{\prime}-x_{y 2}^{\prime} \\
x_{y 2}^{\prime}-x_{y 3}^{\prime}
\end{array}\right)^{\top}\right] \\
& =\left(\begin{array}{ll}
\sigma_{11}+\sigma_{22}-\sigma_{12}-\sigma_{21} & \sigma_{12}+\sigma_{23}-\sigma_{22}-\sigma_{13} \\
\sigma_{21}+\sigma_{32}-\sigma_{22}-\sigma_{31} & \sigma_{22}+\sigma_{33}-\sigma_{23}-\sigma_{32}
\end{array}\right) .
\end{aligned}
$$

This provides four equations for the nine terms that need to be estimated. Recognising that weather events are unpredictable beyond a week or two, another common assumption is that the intraseasonal components are uncorrelated if they are a month or more apart, that is, $\sigma_{13}=\sigma_{31}=0[1,2]$. Thus, three additional equations would be needed to estimate all the terms in equation (4). As discussed in the introduction, this is possible when the climate variables are assumed to be AR1 [1,2]. 


\subsection{Estimating the intermonth covariance with rainfall}

This section aims to elucidate the covarying relationship between the intraseasonal component of the atmospheric pressure field and rainfall, both within each month and between adjacent months. It is assumed that for each month there exists daily rainfall data $R_{y m, t}$ and pressure data $x_{y m, t}$ with $t \in\left(0, T_{m}\right)$ and $T_{m}$, the number of days in month $m$. It is also assumed that $\sigma_{13}=\sigma_{31}=0$. Then, by definition the intermonth covariance

$$
\begin{aligned}
\sigma_{m n} & \equiv E\left[\left(\frac{1}{T_{m}} \sum_{s=1}^{T_{m}} \varepsilon_{y m, s}\right)\left(\frac{1}{T_{n}} \sum_{t=1}^{T_{n}}\left(R_{y n, t}-E\left[R_{y n, t}\right]\right)\right)\right] \\
& =E\left[\left(\frac{1}{T_{m}} \sum_{s=1}^{T_{m}} \varepsilon_{y m, s}\right)\left(\frac{1}{T_{n}} \sum_{t=1}^{T_{n}} R_{y n, t}\right)\right],
\end{aligned}
$$

using the assumption that $E\left[\varepsilon_{y m, s}\right]=0$ (as in equation (3)), and $T_{n}$ is the days in month $n$. The problem then reduces to estimating $E\left[\varepsilon_{y m, s} R_{y n, t}\right]$.

Here, we use a stochastic, two state, first order, Markov chain model [9] fitted to daily precipitation data to estimate the intermonth correlation between monthly means of the precipitation during a season. Let $\left\{J_{m, t}, t=1, \ldots, T_{m}\right\}$ denote the sequence of daily precipitation occurrences in month $\mathrm{m}$ with $T_{m}$ days such that $J_{m, t}=1$ indicates a 'wet day', defined here as a day with at least $1 \mathrm{~mm}$ of rain, and $J_{\mathrm{m}, \mathrm{t}}=0$ a 'dry day'. A first order, Markov chain model is completely characterised by the transition probability

$$
P_{m, j k}=\operatorname{Pr}\left(J_{m,(t+1)}=k \mid J_{m, t}=j\right),
$$

where $j, k=0,1$. The transition probabilities are estimated for each month using daily data over all years, and hence we construct a one step transition matrix $\mathbf{P}_{m}$ for month $\mathbf{m}$,

$$
\mathbf{P}_{\mathrm{m}}=\left(\begin{array}{ll}
\mathrm{P}_{\mathrm{m}, 00} & \mathrm{P}_{\mathrm{m}, 01} \\
\mathrm{P}_{\mathrm{m}, 10} & \mathrm{P}_{\mathrm{m}, 11}
\end{array}\right) .
$$


Then the t-step transition probability is simply $\mathbf{P}_{\mathfrak{m}}^{(\mathrm{t})}=\mathbf{P}_{\mathrm{m}}^{(\mathrm{t}-1)} \mathbf{P}_{\mathfrak{m}}$, and Feller [10] showed that

$$
\begin{aligned}
\mathbf{P}_{m}^{(t)}= & \frac{1}{P_{m, 10}+P_{m, 01}}\left(\begin{array}{ll}
P_{m, 10} & P_{m, 01} \\
P_{m, 10} & P_{m, 01}
\end{array}\right) \\
& +\frac{\left(1-P_{m, 10}-P_{m, 01}\right)^{t}}{P_{m, 10}+P_{m, 01}}\left(\begin{array}{cc}
P_{m, 01} & -P_{m, 01} \\
-P_{m, 10} & P_{m, 10}
\end{array}\right) .
\end{aligned}
$$

Suppose now that $R_{y m, t}$ and the intensity of rainfall on wet days $\left(R_{y m, t}>0\right.$, or $J_{m, t}=1$ ) are conditionally independent and identically distributed with respect to time $\boldsymbol{t}[7,8]$. Then, for any wet day in month $\mathfrak{m}$, the first moment of the intensity $\eta_{m}$ is estimated from daily data as

$$
\eta_{m} \equiv E\left[R_{y m, t} \mid J_{m, t}=1\right]
$$

The stationary probability of rainfall $\pi_{\mathrm{m}}$ [9] is estimated by

$$
\pi_{\mathrm{m}} \equiv \operatorname{Pr}\left(J_{\mathrm{m}, \mathrm{t}}=1\right)=\frac{\mathrm{P}_{\mathrm{m}, 01}}{\left(1-\mathrm{P}_{\mathrm{m}, 11}+\mathrm{P}_{\mathrm{m}, 01}\right)} .
$$

Also, for days in months $m$ and $n$, and $k, j \in(0,1)$, transition probabilities are calculated as

$$
\operatorname{Pr}\left(J_{n, t}=k \mid J_{m, s}=j\right)= \begin{cases}\mathbf{P}_{m}^{(t-s)}[j+1, k+1], & n=m, t>s, \\ \mathbf{P}_{m}^{\left(T_{m}-s\right)} \mathbf{P}_{n}^{(t)}[j+1, k+1], & n=m+1 .\end{cases}
$$

To estimateE $\left[\varepsilon_{y m, s} R_{y n, t}\right]$, it is assumed that $\varepsilon_{y m, s}$ depends only on the rainfall occurrence $J_{m, s}$, that is, it is conditionally independent of the set $\left\{R_{y n, t}\right\}$, given $\mathrm{J}_{\mathrm{m}, \mathrm{s}}$. Three cases need to be considered.

1. $m<n$ :

$$
\begin{aligned}
& \mathrm{E}\left[\varepsilon_{y m, s} R_{y n, t}\right] \\
& =E\left[\varepsilon_{y m, s} R_{y n, t} \mid J_{m, s}=1\right] \operatorname{Pr}\left(J_{m, s}=1\right)
\end{aligned}
$$




$$
\begin{aligned}
& +\mathrm{E}\left[\varepsilon_{y m, s} R_{y n, t} \mid J_{m, s}=0\right] \operatorname{Pr}\left(J_{m, s}=0\right) \\
= & \pi_{m} \operatorname{Pr}\left(J_{n, t}=1 \mid J_{m, s}=1\right) E\left[\varepsilon_{y m, s} R_{y n, t} \mid J_{m, s}=1, J_{n, t}=1\right] \\
& +\left(1-\pi_{m}\right) \operatorname{Pr}\left(J_{n, t}=1 \mid J_{m, s}=0\right) E\left[\varepsilon_{y m, s} R_{y n, t} \mid J_{m, s}=0, J_{n, t}=1\right] \\
= & \pi_{m} \operatorname{Pr}\left(J_{n, t}=1 \mid J_{m, s}=1\right) E\left[\varepsilon_{y m, s} \mid J_{m, s}=1\right] E\left[R_{y n, t} \mid J_{n, t}=1\right] \\
& +\left(1-\pi_{m}\right) \operatorname{Pr}\left(J_{n, t}=1 \mid J_{m, s}=0\right) E\left[\varepsilon_{y m, s} \mid J_{m, s}=0\right] E\left[R_{y n, t} \mid J_{n, t}=1\right] \\
= & \eta_{m}\left\{\pi_{m} \operatorname{Pr}\left(J_{n, t}=1 \mid J_{m, s}=1\right) \lambda_{m, 1}\right. \\
& \left.+\left(1-\pi_{m}\right) \operatorname{Pr}\left(J_{n, t}=1 \mid J_{m, s}=0\right) \lambda_{m, o}\right\}
\end{aligned}
$$

where $\lambda_{\mathrm{m}, 1}=\mathrm{E}\left[\varepsilon_{y m, s} \mid J_{m, s}=1\right]$ and $\lambda_{m, 0}=E\left[\varepsilon_{y m, s} \mid J_{m, s}=0\right]$ are the only terms that need to be estimated.

2. $m>n$ :

$$
\begin{aligned}
\mathrm{E}[ & \left.\varepsilon_{y m, s} R_{y n, t}\right] \\
= & \mathrm{E}\left[\varepsilon_{y m, s} R_{y n, t} \mid J_{n, t}=1\right] \operatorname{Pr}\left(J_{n, t}=1\right) \\
= & \pi_{n} \operatorname{Pr}\left(J_{m, s}=1 \mid J_{n, t}=1\right) \mathrm{E}\left[\varepsilon_{y m, s} R_{y n, t} \mid J_{m, s}=1, J_{n, t}=1\right] \\
& +\pi_{n} \operatorname{Pr}\left(J_{m, s}=0 \mid J_{n, t}=1\right) \mathrm{E}\left[\varepsilon_{y m, s} R_{y n, t} \mid J_{m, s}=0, J_{n, t}=1\right] \\
= & \pi_{n} \operatorname{Pr}\left(J_{m, s}=1 \mid J_{n, t}=1\right) \mathrm{E}\left[\varepsilon_{y m, s} \mid J_{m, s}=1\right] \mathrm{E}\left[R_{y n, t} \mid J_{n, t}=1\right] \\
& +\pi_{n} \operatorname{Pr}\left(J_{m, s}=0 \mid J_{n, t}=1\right) \mathrm{E}\left[\varepsilon_{y m, s} \mid J_{m, s}=0\right] \mathrm{E}\left[R_{y n, t} \mid J_{n, t}=1\right] \\
= & \eta_{n} \pi_{n}\left\{\operatorname{Pr}\left(J_{m, s}=1 \mid J_{n, t}=1\right) \lambda_{m, 1}+\operatorname{Pr}\left(J_{m, s}=0 \mid J_{n, t}=1\right) \lambda_{m, 0}\right\} .
\end{aligned}
$$

3. $m=n:$

(a) If $s<t$, then use equation (13) with $\mathrm{m}=\mathrm{n}$.

(b) If $s>t$, then use equation (14) with $\mathrm{m}=\mathrm{n}$.

(c) If $s=t$, then

$$
\begin{aligned}
\mathrm{E}\left[\varepsilon_{y m, s} R_{y m, s}\right] & =\mathrm{E}\left[\varepsilon_{y m, s} R_{y m, s} \mid J_{m, s}=1\right] \operatorname{Pr}\left(J_{m, s}=1\right) \\
& =\pi_{m} \mathrm{E}\left[\varepsilon_{y m, s} \mid J_{m, s}=1\right] \mathrm{E}\left[R_{y m, s} \mid J_{m, s}=1\right] \\
& =\eta_{m} \pi_{m} \lambda_{m, 1} .
\end{aligned}
$$


The problem now reduces to estimating $\lambda_{m, 1}$ and $\lambda_{m, 0}$. We define

$$
a_{m} \equiv \frac{1}{Y} \sum_{y=1}^{Y} \frac{1}{N_{m}(y)} \sum_{t, J_{m, t}-1}\left(x_{y m, t+1}-x_{y m, t}\right) \approx E\left[\varepsilon_{y m, t+1}-\varepsilon_{y m, t} \mid J_{m, t}=1\right],
$$

where $N_{m}(y)$ is the number of days within month $m$ when $J_{m, t}=1$ in year $y$, and $a_{m}$ is calculated from the daily data. Since $J_{m, t}$ is a stationary binary Markov chain within the month,

$$
\begin{aligned}
\mathrm{E}\left[\varepsilon_{y m, t+1} \mid J_{m, t}=1\right]= & \operatorname{Pr}\left(J_{\mathfrak{m}, \mathrm{t}+1}=0 \mid J_{\mathfrak{m}, \mathrm{t}}=1\right) \mathrm{E}\left[\varepsilon_{y m, t+1} \mid J_{m, t+1}=0\right] \\
& +\operatorname{Pr}\left(J_{\mathfrak{m}, \mathrm{t}+1}=1 \mid J_{\mathfrak{m}, \mathrm{t}}=1\right) \mathrm{E}\left[\varepsilon_{y m, t+1} \mid J_{m, t+1}=1\right] \\
= & P_{\mathfrak{m}, 11} \lambda_{\mathfrak{m}, 1}+P_{m, 10} \lambda_{m, 0} .
\end{aligned}
$$

That is,

$$
a_{m}=P_{m, 11} \lambda_{m, 1}+P_{m, 10} \lambda_{m, 0}-\lambda_{m, 1},
$$

since $P_{m, 11}=1-P_{m, 10}$. Also, $E\left[\varepsilon_{y m, t}\right]=0$ implies that

$$
\begin{aligned}
E\left[\varepsilon_{y m, t}\right] & =\operatorname{Pr}\left(J_{m, t}=0\right) E\left[\varepsilon_{y m, \tau} \mid J_{m, t}=0\right]+\operatorname{Pr}\left(J_{m, t}=1\right) E\left[\varepsilon_{y m, \tau} \mid J_{m, t}=1\right] \\
& =\frac{P_{m, 01}}{P_{m, 10}+P_{m, 01}} \lambda_{m, 1}+\frac{P_{m, 10}}{P_{m, 10}+P_{m, 01}} \lambda_{m, 0}=0 .
\end{aligned}
$$

Hence, using equations (18) and (19),

$$
\begin{aligned}
& \lambda_{m, 1}=-\frac{a_{m}}{\left(P_{m, 10}+P_{m, 01}\right)}, \\
& \lambda_{m, 0}=a_{m} \frac{P_{m, 01}}{P_{m, 10}\left(P_{m, 10}+P_{m, 01}\right)},
\end{aligned}
$$

and the covariance $\sigma_{\mathfrak{m} n}$ (equation (6)) can be estimated.

\section{Example}

To illustrate the methodology, it has been applied to daily Australian rainfall (mm/day) and Southern Hemisphere geopotential height $(\mathrm{m})$ data on 
the $500 \mathrm{hPa}$ pressure level for March, April, May (MAM) from 1958 to 2006. The rainfall data is from the Australian Bureau of Meteorology National Climate Centre gridded historical dataset [11]. The height data is from the National Centers for Environmental Prediction (NCEP) and National Center for Atmospheric Research (NCAR) re-analyses [12]. The rainfall is on a $1^{\circ} \times 1^{\circ}$ latitude/longitude grid, and the geopotential height on a $2.5^{\circ} \times 2.5^{\circ}$ grid. For each rainfall grid point, the intermonth covariance $\sigma_{m n}(m \neq n)$ and within-month covariance $(m=n)$ were calculated for all height gridpoints.

Figure 1 shows the covariances for rainfall at $\left(116^{\circ} \mathrm{E}, 33.5^{\circ} \mathrm{S}\right)$ in the far southwest of Western Australia. In all cases, the pattern of covariances consists of a hemispheric wavetrain of alternating sign largely confined between $20^{\circ} \mathrm{S}$ and $60^{\circ} \mathrm{S}$ with wavenumber between 5 and 6 . These patterns are reminiscent of the typical daily weather patterns in the height field during this season. The largest magnitude of the covariance occurs in the vicinity of the rainfall location. Over the region of the rainfall, the within-month covariances (Figure $1(\mathrm{a})-(\mathrm{c})$ ) are very negative, indicating an association with low pressure systems, as might be expected. When the height field leads (lags) the rainfall, the intermonth covariances (Figure 1(d)-(g)), are negative (positive) over this region, and are associated with low (high) pressure anomalies. However, as indicated by the different contour scaling, the within-month covariances are one or two orders of magnitude larger than the intermonth covariances.

Figure 2 shows the corresponding results for rainfall at $\left(152^{\circ} \mathrm{E}, 27.5^{\circ} \mathrm{S}\right)$ on the central eastern coast of Australia. In this case, the patterns of covariance consist of a wavetrain over the eastern part of Australia arcing poleward into the Pacific Ocean and then equatorward over South America and into the Atlantic and Indian Ocean. This pattern is reminiscent of weather systems associated with intraseasonal variability. Quite negative covariances occur either over, or slightly upstream, of the region of rainfall. Again, the within-month covariances are one or two orders of magnitude larger than the intermonth covariances.

It is not possible to show the patterns of covariation between the height field 
and rainfall at all locations, but they all share the common property that they are reminiscent of weather systems, and in all cases, the within-month covariances are at least one order, or typically two orders, of magnitude larger than the intermonth covariances.

\section{Conclusions}

A methodology has been formulated that allows the within-month and intermonth covariances between the intraseasonal component of an atmospheric pressure field (geopotential height) and rainfall to be estimated using daily data. The method produces patterns of covariability for a particular rainfall location, and these resemble typical weather patterns associated with the location. The within-month covariances are generally much larger than the intermonth covariances. This suggests that the intermonth covariances make relatively little, or negligible, contribution to the interannual covariance of the seasonal mean of the intrasasonal components of height and rainfall (equation (4)). That is, a reasonably accurate estimate for this term is

$$
\mathrm{V}\left(\epsilon_{\mathrm{yo}}, \epsilon_{\mathrm{yo}}^{\prime}\right)=\frac{1}{9}\left(\sigma_{11}+\sigma_{22}+\sigma_{33}\right)
$$

In that case, equation (5) would provide sufficient conditions for an estimate of the within-month covariances using only monthly data.

Acknowledgements S. Grainger is supported by the Australian Climate Change Science Program of the Australian Department of Climate Change and Energy Efficiency. X. Zheng is supported by 2010CB428402 National Program on Key Basic Research Project of China and 40975062/40875062 NSFC. 

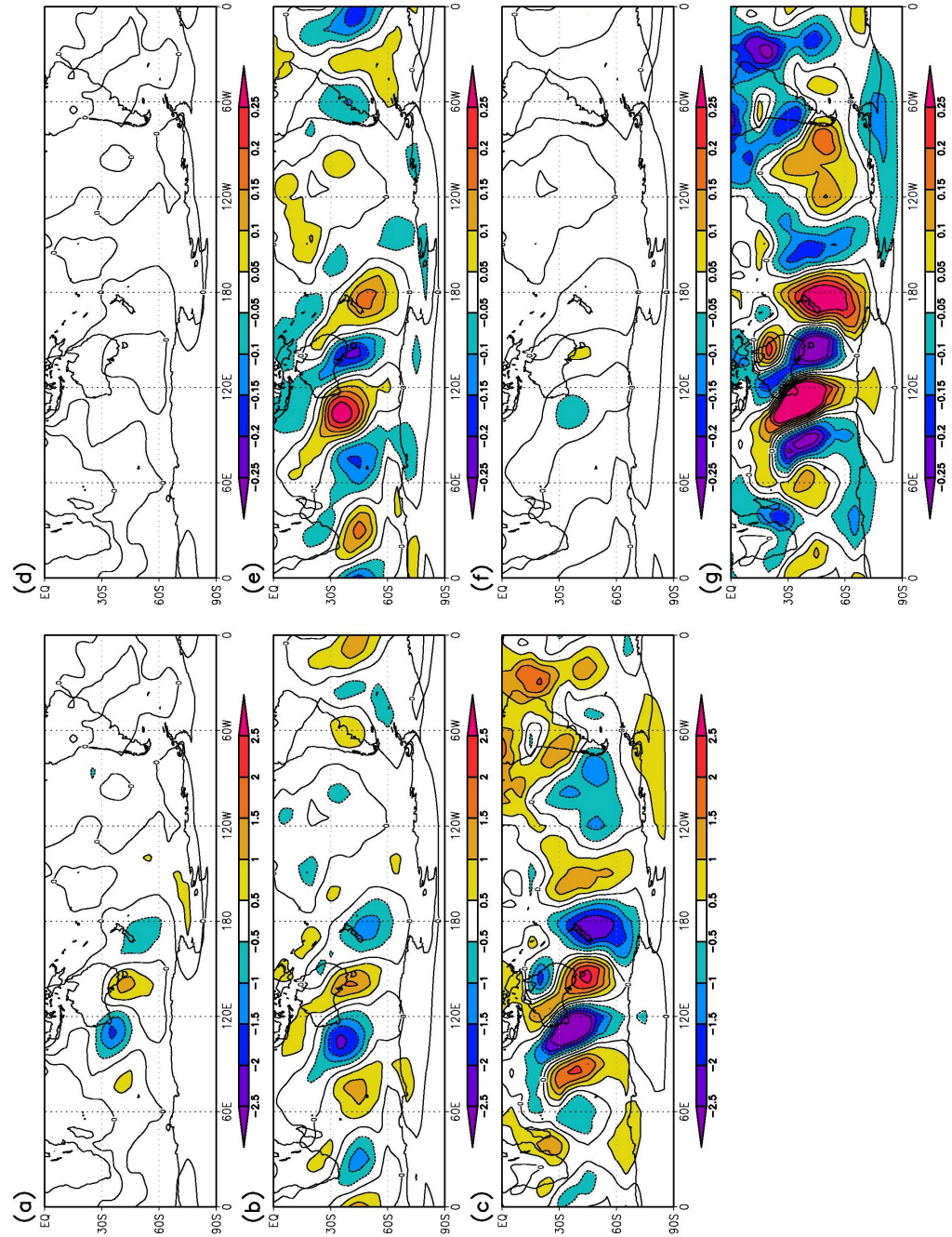

FigURE 1: (a)-(c) Within-month covariances $\sigma_{11}, \sigma_{22}$ and $\sigma_{33}$, respectively; (d)-(g) intermonth covariances $\sigma_{12}, \sigma_{21}, \sigma_{23}$ and $\sigma_{32}$, respectively, for rainfall at $\left(116^{\circ} \mathrm{E}, 33.5^{\circ} \mathrm{s}\right)$. Units are $\mathrm{mm} /$ day $\times \mathrm{m}$ and the covariances have been scaled by 100 . 

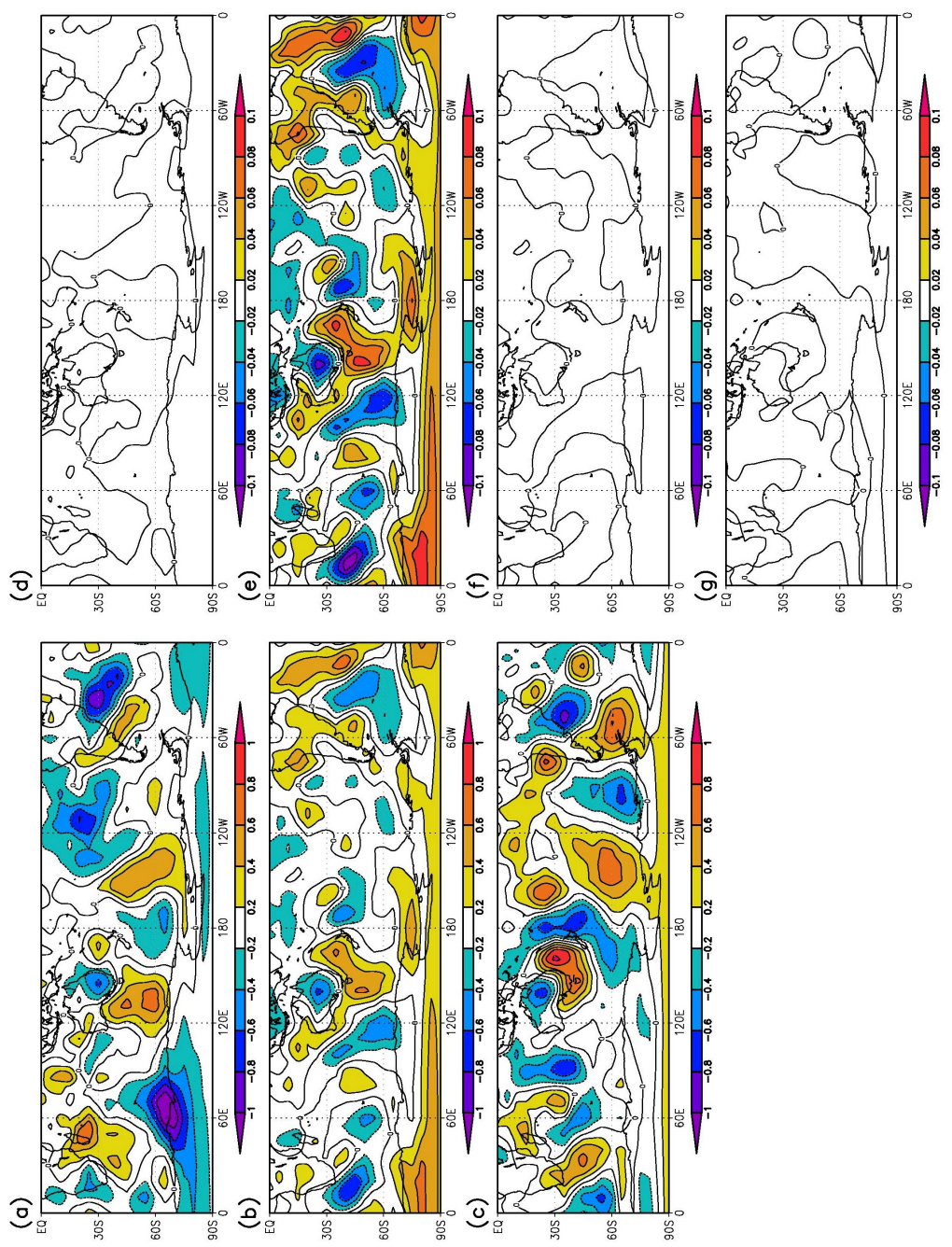

Figure 2: As in Figure 1 but for rainfall at $\left(152^{\circ} \mathrm{E}, 27 \cdot 5^{\circ} \mathrm{S}\right)$. 


\section{References}

[1] C. S. Frederiksen and X. Zheng. Coherent Structures of Interannual Variability of the Atmospheric Circulation: The Role of Intraseasonal Variability. Frontiers in Turbulence and Coherent Structures, World Scientific Lecture Notes in Complex Systems, Vol. 6, Eds Jim Denier and Jorgen Frederiksen, World Scientific Publications, 87-120, 2007. C191, C192, C193, C194

[2] X. Zheng and C. S. Frederiksen. Variability of seasonal-mean fields arising from intraseasonal variability. Part 1, methodology. Climate Dynamics, 23:177-191, 2004. doi:10.1007/s00382-004-0428-7 C191, C192, C193, C194

[3] C. S. Frederiksen and X. Zheng. A Method for constructing skilful seasonal forecasts using slow modes of climate variability. ANZIAM J., 48:C89-C103, 2007. http://anziamj .austms.org.au/ojs/index. php/ANZIAMJ/article/view/114 C191

[4] X. Zheng and C. S. Frederiksen. A study of predictable patterns for seasonal forecasting of New Zealand rainfall. J. Climate, 19:3320-3333, 2006. doi:10.1175/JCLI3798.1 C191, C192

[5] X. Zheng and C. S. Frederiksen. Statistical Prediction of Seasonal Mean Southern Hemisphere 500hPa Geopotential Heights. J. Climate, 20:2791-2809, 2006. doi:10.1175/JCLI4180.1 C191, C192

[6] C. S. Frederiksen and X. Zheng. A Method for extracting coupled patterns of predictable and chaotic components in pairs of climate variables. ANZIAM J., 46:C276-C289, 2005. http://anziamj .austms . org.au/ojs/index.php/ANZIAMJ/article/view/959 C192

[7] C. S. Frederiksen, S. Grainger and X. Zheng. A Method for estimating the potential long-range predictability of precipitation over Western 
Australia. ANZIAM J., 2008. 50:C569-C583, http://anziamj .austms . org.au/ojs/index.php/ANZIAMJ/article/view/1411 C192, C196

[8] R. W. Katz and X. Zheng. Mixture Model For Overdispersion of Precipitation. J. Climate, 12:2528-2537, 1999. doi:10.1175/1520-0442(1999)012ز2528:MMFOOP ¿2.0.CO;2 C196

[9] D. S. Wilks. Statistical Methods in the Atmospheric Sciences. (second edition). Academic Press, 627pp, 2006. C195, C196

[10] W. Feller. An Introduction to Probability Theory and Its Applications. Vol. 2. John Wiley and Sons, 626pp, 1966. C196

[11] D. A. Jones and G. Weymouth. An Australian monthly rainfall data set. Technical Report No. 70, Bur. Met. Australia, 1997. C199

[12] E. Kalnay, M. Kanamitsu, R. Kistler, W. Collins, D. Deaven, L. Gandin, M. Iredell, S. Saha, G. White, J. Woollen, Y. Zhu, M. Chelliah, W. Ebisuzaki, W. Higgins, J. Janowiak, K. C. Mo, C. Ropelewski, J. Wang, A. Leetmaa, R. Reynolds, Roy Jenne, and D. Joseph. The NCEP/NCAR 40-year reanalysis project. Bull. Amer. Meteor. Soc., $77: 437-471,1996$.

doi:10.1175/1520-0477(1996)077;0437:TNYRP ¿2.0.CO;2 C199

\section{Author addresses}

1. C. S. Frederiksen, Centre for Australian Weather and Atmospheric Research, Bureau of Meteorology, Melbourne, Victoria 3001, Australia. mailto:c.frederiksen@bom.gov . au

2. X. Zheng, College of Global Change and Earth System Science, Beijing Normal University, Beijing, China.

3. S. Grainger, Centre for Australian Weather and Atmospheric 
Research, Bureau of Meteorology, Melbourne, Victoria 3001, Australia. 